\title{
Positive solutions of $m$-point integral boundary value problems for second-order $p$-Laplacian dynamic equations on time scales
}

\section{Phollakrit Thiramanus and Jessada Tariboon *}

"Correspondence:

jessadat@kmutnb.ac.th Department of Mathematics, Faculty of Applied Science, King Mongkut's University of Technology North Bangkok, Bangkok, 10800, Thailand

\begin{abstract}
In this article, we use the Krasnosel'skii fixed point theorem, the Avery-Henderson fixed point theorem and the Leggett-Williams fixed point theorem to obtain some results for the existence of at least one, two or three positive solutions of $m$-point integral boundary value problems for nonlinear second-order $p$-Laplacian dynamic equations on time scales. Two examples are presented to illustrate the applications of the results.
\end{abstract}

MSC: 34B15; 34N05

Keywords: positive solution; $p$-Laplacian; time scales; fixed point theorem; integral boundary condition

\section{Introduction}

Analysis on measure chains was initiated by Stefan Hilger [1] as a bridge between continuous and discrete calculus. Dynamic equations on time scales have been a component of applied analysis on measure chains to describe the processes that feature both continuous and discrete elements [2-6]. This subject not only gives a unified approach to the study of differential and difference equations, but also gives an extended approach to the study of dynamic equations with nonuniform step size or a combination of real and discrete domains. Further, the study of time scale equations has led to several important applications, e.g., in the study of economics, insect population models, heat transfer, stock market and epidemic models (see [7-10]), etc. Integral boundary value problems occur in the study of nonlocal phenomena in many different areas of applied mathematics, physics and engineering, e.g., in heat conduction, chemical engineering, underground water flow, thermo-elasticity, plasma physics, etc. (see [11-15] and the references therein).

Throughout this paper, we denote the one-dimensional $p$-Laplacian operator by $\varphi_{p}(u)$, i.e., $\varphi_{p}(u)=|u|^{p-2} u$ for $p>1$ with $\varphi_{p}^{-1}=\varphi_{q}$, where $1 / p+1 / q=1$. For convenience, we make the blanket assumption that $0, T$ are points in a time scale $\mathbb{T}$; for an interval $(0, T)_{\mathbb{T}}$, we always mean $(0, T) \cap \mathbb{T}$. Other types of an interval are defined similarly.

In 2007, Sun and Li [16] discussed the existence of at least one, two or three positive solutions of the following boundary value problem:

$$
\begin{aligned}
& \left(\varphi_{p}\left(u^{\Delta}(t)\right)\right)^{\triangle}+h(t) f\left(u^{\sigma}(t)\right)=0, \quad t \in[a, b]_{\mathbb{T}}, \\
& u(a)-B_{0}\left(u^{\Delta}(a)\right)=0, \quad u^{\Delta}(\sigma(b))=0 .
\end{aligned}
$$


They used the Krasnosel'skii fixed point theorem, the Avery-Henderson fixed point theorem and the Leggett-Williams fixed point theorem to prove the existence of multiple positive solutions to problem (1.1)-(1.2).

In 2009, Zhang and Qiao [17] studied the existence criteria for the $m$-point boundary value problem:

$$
\begin{aligned}
& \left(\varphi_{p}\left(u^{\Delta}(t)\right)\right)^{\Delta}+a(t) f(t, u(t))=0, \quad t \in[0,1]_{\mathbb{T}}, \\
& u(0)=0, \quad u(1)=\sum_{i=1}^{m-2} a_{i} u\left(\xi_{i}\right) .
\end{aligned}
$$

They obtained some results for the existence of multiple positive solutions of problem (1.3)-(1.4) by using the Krasnosel'skii fixed point theorem, the Avery-Henderson fixed point theorem and the Leggett-Williams fixed point theorem.

In 2011, Li and Zhang [18] considered the existence of at least three positive solutions for the boundary value problem with integral boundary conditions:

$$
\begin{aligned}
& \left(\varphi_{p}\left(x^{\Delta}(t)\right)\right)^{\nabla}+\lambda f\left(t, x(t), x^{\Delta}(t)\right)=0, \quad t \in(0, T)_{\mathbb{T}}, \\
& x^{\Delta}(0)=0, \quad \alpha x(T)-\beta x(0)=\int_{0}^{T} g(s) x(s) \nabla s .
\end{aligned}
$$

They established some sufficient conditions for the existence of positive solutions to problem (1.5)-(1.6) by using the Legget-Williams fixed point theorem. For some recent results on the existence of positive solutions for $p$-Laplacian dynamic equations on time scales, see [19-27]. However, to the best of the authors' knowledge, existence results for positive solutions of $m$-point integral boundary value problems for nonlinear $p$-Laplacian dynamic equations on time scales have not been studied.

In this article, we are concerned with the existence of multiple positive solutions to the $m$-point integral boundary value problem for a second-order $p$-Laplacian dynamic equation on time scale $\mathbb{T}$ :

$$
\begin{aligned}
& \left(\varphi_{p}\left(u^{\Delta}(t)\right)\right)^{\Delta}+a(t) f(t, u(t))=0, \quad t \in[0,1]_{\mathbb{T}}, \\
& u^{\Delta}(0)=0, \quad u(1)=\sum_{i=1}^{m-1} \alpha_{i} \int_{\xi_{i-1}}^{\xi_{i}} u(s) \Delta s
\end{aligned}
$$

where $\mathbb{T}$ is a time scale, $0=\xi_{0}<\xi_{1}<\xi_{2}<\cdots<\xi_{m-2}<\xi_{m-1}=1$ and

$\left(\mathrm{H}_{1}\right) \quad 0<\sum_{i=1}^{m-1} \alpha_{i}\left(\xi_{i}-\xi_{i-1}\right)<1$ such that $\alpha_{i} \geq 0$ for $i \in\{1,2, \ldots, m-3\} \cup\{m-1\}, \alpha_{m-2}>0$;

$\left(\mathrm{H}_{2}\right) f \in C_{r d}\left([0,1]_{\mathbb{T}} \times[0, \infty),[0, \infty)\right)$;

$\left(\mathrm{H}_{3}\right) a \in C_{r d}\left([0,1]_{\mathbb{T}},[0, \infty)\right)$ and there exists $t_{0} \in\left(\xi_{m-2}, 1\right)_{\mathbb{T}}$ such that $a\left(t_{0}\right)>0$.

The rest of the paper is organized as follows. In Section 2, we state and prove some lemmas which are used later. In Section 3, we use the Krasnosel'skii [28] fixed point theorem to obtain the existence of at least one positive solution of problem (1.7)-(1.8). In Section 4, by using the Avery-Henderson [29] fixed point theorem, we establish sufficient conditions for the existence of at least two positive solutions of problem (1.7)-(1.8). In Section 5, 
the existence of at least three positive solutions of problem (1.7)-(1.8) are proved by using the Leggett-Williams [30] fixed point theorem. Two illustrative examples are given in Section 6.

For convenience, we list the following well-known definitions which can be found in [4] and the references therein.

Definition 1.1 A time scale $\mathbb{T}$ is an arbitrary nonempty closed subset of the real set $\mathbb{R}$ with topology and ordering inherited from $\mathbb{R}$.

The forward and backward jump operators $\sigma, \rho: \mathbb{T} \rightarrow \mathbb{T}$ and the graininess $\mu: \mathbb{T} \rightarrow \mathbb{R}^{+}$ are defined, respectively, by

$$
\sigma(t):=\inf \{s \in \mathbb{T} \mid s>t\}, \quad \rho(t):=\sup \{s \in \mathbb{T} \mid s<t\}, \quad \mu(t):=\sigma(t)-t,
$$

for all $t \in \mathbb{T}$. If $\sigma(t)>t, t$ is said to be right scattered, and if $\rho(t)<t, t$ is said to be left scattered; if $\sigma(t)=t, t$ is said to be right dense, and if $\rho(t)=t, t$ is said to be left dense. If $\mathbb{T}$ has a left-scattered maximum $M$, define $\mathbb{T}^{k}=\mathbb{T}-\{M\}$; otherwise set $\mathbb{T}^{k}=\mathbb{T}$.

Definition 1.2 A function $f: \mathbb{T} \rightarrow \mathbb{R}$ is rd-continuous (rd-continuous is short for rightdense continuous) provided it is continuous at each right-dense point in $\mathbb{T}$ and has a leftsided limit at each left-dense point in $\mathbb{T}$. The set of rd-continuous functions $f: \mathbb{T} \rightarrow \mathbb{R}$ will be denoted by $C_{r d}(\mathbb{T})=C_{r d}(\mathbb{T}, \mathbb{R})$.

Definition 1.3 For $f: \mathbb{T} \rightarrow \mathbb{R}$ and $t \in \mathbb{T}^{k}$, the delta derivative of $f$ at the point $t$ is defined to be the number $f^{\Delta}(t)$ (provided it exists), with the property that for each $\epsilon>0$, there is a neighborhood $U$ of $t$ such that

$$
\left|f(\sigma(t))-f(s)-f^{\Delta}(t)(\sigma(t)-s)\right| \leq \epsilon|\sigma(t)-s|
$$

for all $s \in U$.

Definition 1.4 For a function $f: \mathbb{T} \rightarrow \mathbb{R}$, the delta derivative is defined at the point $t$ by

$$
f^{\Delta}(t)=\frac{f(\sigma(t))-f(t)}{\sigma(t)-t},
$$

if $f$ is continuous at $t$ and $t$ is right-scattered. If $t$ is not right-scattered, then the derivative is defined by

$$
f^{\Delta}(t)=\lim _{s \rightarrow t} \frac{f(\sigma(t))-f(s)}{\sigma(t)-s}=\lim _{s \rightarrow t} \frac{f(t)-f(s)}{t-s}
$$

provided this limit exists.

Definition 1.5 If $F^{\Delta}(t)=f(t)$, then we define the delta integral by

$$
\int_{a}^{t} f(s) \Delta s=F(t)-F(a)
$$




\section{Preliminaries}

In this section, we first prove and recall some lemmas which are used in what follows.

Lemma 2.1 Let $\sum_{i=1}^{m-1} \alpha_{i}\left(\xi_{i}-\xi_{i-1}\right) \neq 1$. Then, for $y \in C_{r d}\left([0,1]_{\mathbb{T}}, \mathbb{R}\right)$, the problem

$$
\begin{aligned}
& \left(\varphi_{p}\left(u^{\Delta}(t)\right)\right)^{\Delta}+y(t)=0, \quad t \in[0,1]_{\mathbb{T}}, \\
& u^{\Delta}(0)=0, \quad u(1)=\sum_{i=1}^{m-1} \alpha_{i} \int_{\xi_{i-1}}^{\xi_{i}} u(s) \Delta s,
\end{aligned}
$$

has a unique solution

$$
\begin{aligned}
u(t)= & -\int_{0}^{t} \varphi_{q}\left(\int_{0}^{\tau} y(s) \Delta s\right) \Delta \tau \\
& -\frac{1}{1-\sum_{i=1}^{m-1} \alpha_{i}\left(\xi_{i}-\xi_{i-1}\right)} \sum_{i=1}^{m-1} \alpha_{i} \int_{\xi_{i-1}}^{\xi_{i}} \int_{0}^{\eta} \varphi_{q}\left(\int_{0}^{\tau} y(s) \Delta s\right) \Delta \tau \Delta \eta \\
& +\frac{1}{1-\sum_{i=1}^{m-1} \alpha_{i}\left(\xi_{i}-\xi_{i-1}\right)} \int_{0}^{1} \varphi_{q}\left(\int_{0}^{\tau} y(s) \Delta s\right) \Delta \tau
\end{aligned}
$$

Proof Integrating (2.1) from 0 to $t$ and using the first condition of (2.2), one gets

$$
u^{\Delta}(t)=-\varphi_{q}\left(\int_{0}^{t} y(s) \triangle s\right)
$$

Integrating (2.4) from 0 to $t$, we obtain

$$
u(t)=u(0)-\int_{0}^{t} \varphi_{q}\left(\int_{0}^{\tau} y(s) \Delta s\right) \triangle \tau .
$$

In particular, for $t=1$, we have

$$
u(1)=u(0)-\int_{0}^{1} \varphi_{q}\left(\int_{0}^{\tau} y(s) \Delta s\right) \Delta \tau .
$$

Using the second condition of (2.2), we get that

$$
\begin{aligned}
u(0) & -\int_{0}^{1} \varphi_{q}\left(\int_{0}^{\tau} y(s) \Delta s\right) \Delta \tau \\
& =u(0) \sum_{i=1}^{m-1} \alpha_{i}\left(\xi_{i}-\xi_{i-1}\right)-\sum_{i=1}^{m-1} \alpha_{i} \int_{\xi_{i-1}}^{\xi_{i}} \int_{0}^{\eta} \varphi_{q}\left(\int_{0}^{\tau} y(s) \Delta s\right) \Delta \tau \Delta \eta .
\end{aligned}
$$

Hence,

$$
\begin{aligned}
u(0)= & \frac{1}{1-\sum_{i=1}^{m-1} \alpha_{i}\left(\xi_{i}-\xi_{i-1}\right)}\left[\int_{0}^{1} \varphi_{q}\left(\int_{0}^{\tau} y(s) \triangle s\right) \Delta \tau\right. \\
& \left.-\sum_{i=1}^{m-1} \alpha_{i} \int_{\xi_{i-1}}^{\xi_{i}} \int_{0}^{\eta} \varphi_{q}\left(\int_{0}^{\tau} y(s) \triangle s\right) \triangle \tau \Delta \eta\right] .
\end{aligned}
$$

Substituting the value of $u(0)$ in (2.5), we obtain the solution (2.3). 
Lemma 2.2 Let $\sum_{i=1}^{m-1} \alpha_{i}\left(\xi_{i}-\xi_{i-1}\right) \neq 1$. If $y \in C_{r d}\left([0,1]_{\mathbb{T}},[0, \infty)\right)$, then the unique solution $u$ of problem (2.1)-(2.2) satisfies

$$
u^{\Delta}(t) \leq 0, \quad u^{\Delta \Delta}(t) \leq 0, \quad t \in[0,1]_{\mathbb{T}} .
$$

Proof From (2.4), we have $u^{\Delta}(t) \leq 0$ for $t \in[0,1]_{\mathbb{T}}$. In fact, $\varphi_{q}(x)$ is a monotone increasing continuously differentiable function and

$$
\left(\int_{0}^{t} y(s) \Delta s\right)^{\Delta}=y(t) \geq 0
$$

Then, by the chain rule [4], we get $u^{\Delta \Delta}(t) \leq 0$ for $t \in[0,1]_{\mathbb{T}}$.

Lemma 2.3 Let $0<\sum_{i=1}^{m-1} \alpha_{i}\left(\xi_{i}-\xi_{i-1}\right)<1$. If $y \in C_{r d}\left([0,1]_{\mathbb{T}},[0, \infty)\right)$, then the unique solution u of problem (2.1)-(2.2) satisfies

$$
u(t) \geq 0, \quad t \in[0,1]_{\mathbb{T}} .
$$

Proof From Lemma 2.2, $u^{\Delta}(t) \leq 0$ for $t \in[0,1]_{\mathbb{T}}$, we know that $u$ is nonincreasing on $[0,1]_{\mathbb{T}}$. Consequently, for each $t_{1}, t_{2} \in \mathbb{T}$ and $t_{1} \leq t_{2}$, it holds that $u\left(t_{1}\right) \geq u\left(t_{2}\right)$.

Therefore,

$$
u(0) \geq u\left(\xi_{1}\right) \geq \cdots \geq u\left(\xi_{i-1}\right) \geq u\left(\xi_{i}\right) \geq \cdots \geq u\left(\xi_{m-2}\right) \geq u(1) .
$$

If $u(1)<0$, then the second condition of (2.2) together with (2.6) implies that

$$
\begin{aligned}
u(1) & =\sum_{i=1}^{m-1} \alpha_{i} \int_{\xi_{i-1}}^{\xi_{i}} u(s) \Delta s \geq \sum_{i=1}^{m-1} \alpha_{i} u\left(\xi_{i}\right)\left(\xi_{i}-\xi_{i-1}\right) \\
& \geq u(1) \sum_{i=1}^{m-1} \alpha_{i}\left(\xi_{i}-\xi_{i-1}\right) .
\end{aligned}
$$

This contradicts the fact that $0<\sum_{i=1}^{m-1} \alpha_{i}\left(\xi_{i}-\xi_{i-1}\right)<1$.

If $u(0)<0$, it follows that $u(1)<0$ since $u$ is nonincreasing. Hence, we get a contradiction. Indeed, if $u(0)<0$ and $u(1)<0$, we again obtain a contradiction.

Lemma 2.4 Let $\sum_{i=1}^{m-1} \alpha_{i}\left(\xi_{i}-\xi_{i-1}\right)>1$. If $y \in C_{r d}\left([0,1]_{\mathbb{T}},[0, \infty)\right)$, then problem (2.1)-(2.2) has no positive solutions.

Proof Suppose that problem (2.1)-(2.2) has a positive solution $u$ satisfying $u(t) \geq 0$ for $t \in[0,1]_{\mathbb{T}}$. Then $u\left(\xi_{i}\right) \geq 0$ for all $i=1, \ldots, m-1$. By the second condition of (2.2) and (2.6), we have

$$
\begin{aligned}
u(1) & =\sum_{i=1}^{m-1} \alpha_{i} \int_{\xi_{i-1}}^{\xi_{i}} u(s) \Delta s \\
& \geq \sum_{i=1}^{m-1} \alpha_{i} u\left(\xi_{i}\right)\left(\xi_{i}-\xi_{i-1}\right)
\end{aligned}
$$




$$
\begin{aligned}
& \geq u(1) \sum_{i=1}^{m-1} \alpha_{i}\left(\xi_{i}-\xi_{i-1}\right) \\
& >u(1),
\end{aligned}
$$

getting a contradiction.

Let $E$ denote the Banach space $C_{r d}[0,1]_{\mathbb{T}}$ with the norm $\|u\|=\sup _{t \in[0,1]_{\mathbb{T}}}|u(t)|$. Define the cone $P \subset E$, by

$$
\begin{gathered}
P=\left\{u \in E \mid u(t) \geq 0, u^{\Delta}(t) \leq 0, u^{\Delta \Delta}(t) \leq 0 \text { for } t \in[0,1]_{\mathbb{T}}\right. \\
\left.\quad \text { and } u^{\Delta}(0)=0, u(1)=\sum_{i=1}^{m-1} \alpha_{i} \int_{\xi_{i-1}}^{\xi_{i}} u(s) \Delta s\right\}
\end{gathered}
$$

Define the operator $A: P \rightarrow E$ by

$$
\begin{aligned}
A u(t)= & -\int_{0}^{t} \varphi_{q}\left(\int_{0}^{\tau} a(s) f(s, u(s)) \Delta s\right) \Delta \tau \\
& -\frac{1}{1-\Lambda} \sum_{i=1}^{m-1} \alpha_{i} \int_{\xi_{i-1}}^{\xi_{i}} \int_{0}^{\eta} \varphi_{q}\left(\int_{0}^{\tau} a(s) f(s, u(s)) \Delta s\right) \Delta \tau \Delta \eta \\
& +\frac{1}{1-\Lambda} \int_{0}^{1} \varphi_{q}\left(\int_{0}^{\tau} a(s) f(s, u(s)) \Delta s\right) \Delta \tau
\end{aligned}
$$

where a positive constant $\Lambda=\sum_{i=1}^{m-1} \alpha_{i}\left(\xi_{i}-\xi_{i-1}\right)<1$. In view of Lemma 2.1, the solutions of problem (1.7)-(1.8) are given by the operator equation, $u(t)=A u(t)$.

From (2.8), we claim that for each $u \in P, A u \in P$ and satisfies (1.8). In fact, for $t \in[0,1]_{\mathbb{T}}$, we get

$$
\begin{aligned}
A u(t) \geq & A u(1) \\
= & -\int_{0}^{1} \varphi_{q}\left(\int_{0}^{\tau} a(s) f(s, u(s)) \Delta s\right) \Delta \tau \\
& -\frac{1}{1-\Lambda} \sum_{i=1}^{m-1} \alpha_{i} \int_{\xi_{i-1}}^{\xi_{i}} \int_{0}^{\eta} \varphi_{q}\left(\int_{0}^{\tau} a(s) f(s, u(s)) \Delta s\right) \Delta \tau \Delta \eta \\
& +\frac{1}{1-\Lambda} \int_{0}^{1} \varphi_{q}\left(\int_{0}^{\tau} a(s) f(s, u(s)) \Delta s\right) \Delta \tau \\
= & \frac{\Lambda}{1-\Lambda} \int_{0}^{1} \varphi_{q}\left(\int_{0}^{\tau} a(s) f(s, u(s)) \Delta s\right) \Delta \tau \\
& -\frac{1}{1-\Lambda} \sum_{i=1}^{m-1} \alpha_{i} \int_{\xi_{i-1}}^{\xi_{i}} \int_{0}^{\eta} \varphi_{q}\left(\int_{0}^{\tau} a(s) f(s, u(s)) \Delta s\right) \Delta \tau \Delta \eta \geq 0 .
\end{aligned}
$$

This implies that $A u(t) \geq 0$ for $t \in[0,1]_{\mathbb{T}}$. As in Lemma 2.2, we can prove that $(A u)^{\Delta}(t) \leq$ $0,(A u)^{\Delta \Delta}(t) \leq 0$ for $t \in[0,1]_{\mathbb{T}}$. In addition, we find that $(A u)^{\Delta}(0)=0$ and $(A u)(1)=$ $\sum_{i=1}^{m-1} \alpha_{i} \int_{\xi_{i-1}}^{\xi_{i}} A u(s) \Delta s$. So, $A: P \rightarrow P$. It is also easy to check that $A: P \rightarrow P$ is completely continuous. 
Lemma 2.5 Let $\left(\mathrm{H}_{1}\right)$ hold. If $u \in P$, then

$$
\min _{t \in[0,1]_{\mathbb{T}}} u(t) \geq \gamma\|u\|
$$

where

$$
\gamma=\frac{\alpha_{m-2}\left(\xi_{m-2}-\xi_{m-3}\right)\left(1-\xi_{m-2}\right)}{1-\alpha_{m-2} \xi_{m-2}\left(\xi_{m-2}-\xi_{m-3}\right)}
$$

which $\gamma>0$.

Proof Since $u^{\Delta}(t) \leq 0$ for $t \in[0,1]_{\mathbb{T}}$, we have $\|u\|=u(0), \min _{t \in[0,1]_{\mathbb{T}}} u(t)=u(1)$.

Thus,

$$
u(1)=\sum_{i=1}^{m-1} \alpha_{i} \int_{\xi_{i-1}}^{\xi_{i}} u(s) \Delta s \geq \sum_{i=1}^{m-1} \alpha_{i} u\left(\xi_{i}\right)\left(\xi_{i}-\xi_{i-1}\right) \geq \alpha_{m-2} u\left(\xi_{m-2}\right)\left(\xi_{m-2}-\xi_{m-3}\right) .
$$

From $u^{\Delta \Delta}(t) \leq 0$ for $t \in[0,1]_{\mathbb{T}}$ and (2.11), we get

$$
\begin{aligned}
u(0) & \leq u(1)+\frac{u(1)-u\left(\xi_{m-2}\right)}{1-\xi_{m-2}}(0-1) \\
& \leq u(1)\left[1-\frac{1}{1-\xi_{m-2}}+\frac{1}{\alpha_{m-2}\left(\xi_{m-2}-\xi_{m-3}\right)\left(1-\xi_{m-2}\right)}\right] \\
& =u(1)\left[\frac{1-\alpha_{m-2} \xi_{m-2}\left(\xi_{m-2}-\xi_{m-3}\right)}{\alpha_{m-2}\left(\xi_{m-2}-\xi_{m-3}\right)\left(1-\xi_{m-2}\right)}\right] .
\end{aligned}
$$

This implies that

$$
\min _{t \in[0,1]_{\mathbb{T}}} u(t) \geq \frac{\alpha_{m-2}\left(\xi_{m-2}-\xi_{m-3}\right)\left(1-\xi_{m-2}\right)}{1-\alpha_{m-2} \xi_{m-2}\left(\xi_{m-2}-\xi_{m-3}\right)}\|u\|
$$

Note that $\left(\mathrm{H}_{1}\right)$ yields

$$
0<1-\sum_{i=1}^{m-1} \alpha_{i}\left(\xi_{i}-\xi_{i-1}\right)<1-\alpha_{m-2}\left(\xi_{m-2}-\xi_{m-3}\right)<1-\alpha_{m-2} \xi_{m-2}\left(\xi_{m-2}-\xi_{m-3}\right) .
$$

Thus we have $\gamma>0$. The proof of Lemma 2.5 is complete.

In the following, for the sake of convenience, we set constants

$$
\begin{aligned}
& L=\frac{1-\Lambda}{\int_{0}^{1} \varphi_{q}\left(\int_{0}^{\tau} a(s) \Delta s\right) \Delta \tau}, \\
& M=\frac{1-\Lambda}{\gamma \xi_{m-2} \int_{\xi_{m-2}}^{1} \varphi_{q}\left(\int_{\xi_{m-2}}^{\tau} a(s) \Delta s\right) \Delta \tau}, \\
& N=\frac{1-\Lambda}{\gamma \Lambda \int_{\xi_{m-2}}^{1} \varphi_{q}\left(\int_{\xi_{m-2}}^{\tau} a(s) \Delta s\right) \Delta \tau} .
\end{aligned}
$$




\section{Existence of at least one positive solution}

Now we are in a position to establish the main result. Our first result is based on the Krasnosel'skii fixed point theorem.

Theorem 3.1 (see [28]) Let E be a Banach space, and let $P \subset E$ be a cone. Assume that $\Omega_{1}$ and $\Omega_{2}$ are bounded open subsets of $E$ with $0 \in \Omega_{1}, \bar{\Omega}_{1} \subset \Omega_{2}$, and let $A: P \cap\left(\bar{\Omega}_{2} \backslash \Omega_{1}\right) \rightarrow P$ be a completely continuous operator such that either

(i) $\|A u\| \leq\|u\|$ for $u \in P \cap \partial \Omega_{1},\|A u\| \geq\|u\|$ for $u \in P \cap \partial \Omega_{2}$; or

(ii) $\|A u\| \geq\|u\|$ for $u \in P \cap \partial \Omega_{1},\|A u\| \leq\|u\|$ for $u \in P \cap \partial \Omega_{2}$ hold.

Then $A$ has a fixed point in $P \cap\left(\bar{\Omega}_{2} \backslash \Omega_{1}\right)$.

Theorem 3.2 Assume that $\left(\mathrm{H}_{1}\right)-\left(\mathrm{H}_{3}\right)$ hold. In addition, suppose that there exist numbers $0<r<R<\infty$ such that

$\left(\mathrm{A}_{1}\right) f(t, u) \leq \varphi_{p}(L) \varphi_{p}(r)$ for $t \in[0,1]_{\mathbb{T}}$ and $0 \leq u \leq r$;

$\left(\mathrm{A}_{2}\right) f(t, u) \geq \varphi_{p}(M r) \varphi_{p}(R)$ for $t \in\left[\xi_{m-2}, 1\right]_{\mathbb{T}}$ and $R \leq u<\infty$,

where constants $L, M$ are defined by (2.12) and (2.13), respectively.

Then problem (1.7)-(1.8) has at least one positive solution.

Proof Firstly, we define a cone $P$ and a completely continuous operator $A: P \rightarrow P$ as in (2.7) and (2.8), respectively.

Let $\Omega_{1}=\left\{u \in C_{r d}\left([0,1]_{\mathbb{T}}\right):\|u\|<r\right\}$. For any $u \in P \cap \partial \Omega_{1}$ with $\|u\|=r$, from condition $\left(\mathrm{A}_{1}\right)$, we obtain

$$
\begin{aligned}
A u(t)= & -\int_{0}^{t} \varphi_{q}\left(\int_{0}^{\tau} a(s) f(s, u(s)) \Delta s\right) \Delta \tau \\
& -\frac{1}{1-\Lambda} \sum_{i=1}^{m-1} \alpha_{i} \int_{\xi_{i-1}}^{\xi_{i}} \int_{0}^{\eta} \varphi_{q}\left(\int_{0}^{\tau} a(s) f(s, u(s)) \Delta s\right) \Delta \tau \Delta \eta \\
& +\frac{1}{1-\Lambda} \int_{0}^{1} \varphi_{q}\left(\int_{0}^{\tau} a(s) f(s, u(s)) \Delta s\right) \Delta \tau \\
\leq & \frac{1}{1-\Lambda} \int_{0}^{1} \varphi_{q}\left(\int_{0}^{\tau} a(s) f(s, u(s)) \Delta s\right) \Delta \tau \\
\leq & \frac{\varphi_{q}\left(\varphi_{p}(L) \varphi_{p}(r)\right)}{1-\Lambda} \int_{0}^{1} \varphi_{q}\left(\int_{0}^{\tau} a(s) \Delta s\right) \Delta \tau \\
= & \frac{r L}{1-\Lambda} \int_{0}^{1} \varphi_{q}\left(\int_{0}^{\tau} a(s) \Delta s\right) \Delta \tau=r=\|u\| .
\end{aligned}
$$

This implies that $\|A u\| \leq\|u\|$ for $u \in P \cap \partial \Omega_{1}$.

Set $\Omega_{2}=\left\{u \in C_{r d}\left([0,1]_{\mathbb{T}}\right):\|u\|<R\right\}$. Since $u \in P \cap \partial \Omega_{2}$, it follows that $\min _{t \in[0,1]_{\mathbb{T}}} u(t) \geq$ $\gamma\|u\|=\gamma R$. Hence from condition $\left(\mathrm{A}_{2}\right)$, for any $u \in P \cap \partial \Omega_{2}$, we have

$$
\begin{aligned}
\|A u\| \geq & A u\left(\xi_{m-2}\right) \\
= & -\int_{0}^{\xi_{m-2}} \varphi_{q}\left(\int_{0}^{\tau} a(s) f(s, u(s)) \Delta s\right) \Delta \tau \\
& -\frac{1}{1-\Lambda} \sum_{i=1}^{m-1} \alpha_{i} \int_{\xi_{i-1}}^{\xi_{i}} \int_{0}^{\eta} \varphi_{q}\left(\int_{0}^{\tau} a(s) f(s, u(s)) \Delta s\right) \Delta \tau \Delta \eta
\end{aligned}
$$




$$
\begin{aligned}
& +\frac{1}{1-\Lambda} \int_{0}^{1} \varphi_{q}\left(\int_{0}^{\tau} a(s) f(s, u(s)) \Delta s\right) \Delta \tau \\
& =\frac{\int_{0}^{1} \varphi_{q}\left(\int_{0}^{\tau} a(s) f(s, u(s)) \Delta s\right) \Delta \tau-\int_{0}^{\xi_{m-2}} \varphi_{q}\left(\int_{0}^{\tau} a(s) f(s, u(s)) \Delta s\right) \Delta \tau}{1-\Lambda} \\
& +\frac{1}{1-\Lambda}\left[\Lambda \int_{0}^{\xi_{m-2}} \varphi_{q}\left(\int_{0}^{\tau} a(s) f(s, u(s)) \Delta s\right) \Delta \tau\right. \\
& \left.-\sum_{i=1}^{m-1} \alpha_{i} \int_{\xi_{i-1}}^{\xi_{i}} \int_{0}^{\eta} \varphi_{q}\left(\int_{0}^{\tau} a(s) f(s, u(s)) \Delta s\right) \Delta \tau \Delta \eta\right] \\
& \geq \frac{1}{1-\Lambda} \int_{\xi_{m-2}}^{1} \varphi_{q}\left(\int_{0}^{\tau} a(s) f(s, u(s)) \Delta s\right) \Delta \tau \\
& -\frac{1}{1-\Lambda} \int_{\xi_{m-2}}^{1} \int_{\xi_{m-2}}^{\eta} \varphi_{q}\left(\int_{0}^{\tau} a(s) f(s, u(s)) \Delta s\right) \Delta \tau \Delta \eta \\
& \geq \frac{1}{1-\Lambda} \int_{\xi_{m-2}}^{1} \varphi_{q}\left(\int_{0}^{\tau} a(s) f(s, u(s)) \Delta s\right) \Delta \tau \\
& -\frac{1}{1-\Lambda} \int_{\xi_{m-2}}^{1} \int_{\xi_{m-2}}^{1} \varphi_{q}\left(\int_{0}^{\tau} a(s) f(s, u(s)) \Delta s\right) \Delta \tau \Delta \eta \\
& =\frac{\xi_{m-2}}{1-\Lambda} \int_{\xi_{m-2}}^{1} \varphi_{q}\left(\int_{0}^{\tau} a(s) f(s, u(s)) \Delta s\right) \Delta \tau \\
& \geq \frac{\xi_{m-2}}{1-\Lambda} \int_{\xi_{m-2}}^{1} \varphi_{q}\left(\int_{\xi_{m-2}}^{\tau} a(s) f(s, u(s)) \Delta s\right) \Delta \tau \\
& \geq \varphi_{q}\left(\varphi_{p}(M \gamma) \varphi_{p}(R)\right) \frac{\xi_{m-2} \int_{\xi_{m-2}}^{1} \varphi_{q}\left(\int_{\xi_{m-2}}^{\tau} a(s) \Delta s\right) \Delta \tau}{1-\Lambda} \\
& =\frac{M \gamma R}{1-\Lambda} \xi_{m-2} \int_{\xi_{m-2}}^{1} \varphi_{q}\left(\int_{\xi_{m-2}}^{\tau} a(s) \Delta s\right) \Delta \tau=\|u\| .
\end{aligned}
$$

Therefore, $\|A u\| \geq\|u\|$ for $u \in P \cap \partial \Omega_{2}$.

Thus, from Theorem 3.1, it follows that $A$ has a fixed point $u$ in $P \cap\left(\bar{\Omega}_{2} \backslash \Omega_{1}\right)$ such that $r \leq\|u\| \leq R$. Therefore, problem (1.7)-(1.8) has at least one positive solution.

\section{Existence of at least two positive solutions}

In this section, we obtain the existence of at least two positive solutions of problem (1.7)-

(1.8) by using the Avery-Henderson fixed point theorem which is as follows.

Theorem 4.1 (see [29]) Let P be a cone in a real Banach space E. Set

$$
P\left(\Phi, \rho_{3}\right)=\left\{u \in P \mid \Phi(u)<\rho_{3}\right\}
$$

Let $v$ and $\Phi$ be increasing nonnegative continuous functionals on $P$, and let $\theta$ be a nonnegative continuous functional on $P$ with $\theta(0)=0$ such that, for some $\rho_{3}>0$ and $N>0$,

$$
\Phi(u) \leq \theta(u) \leq v(u) \quad \text { and } \quad\|u\| \leq N \Phi(u)
$$


for all $u \in \overline{P\left(\Phi, \rho_{3}\right)}$. Suppose there exist a completely continuous operator $A: \overline{P\left(\Phi, \rho_{3}\right)} \rightarrow P$ and $0<\rho_{1}<\rho_{2}<\rho_{3}$ such that

$$
\theta(\lambda u)=\lambda \theta(u) \quad \text { for } 0 \leq \lambda \leq 1 \text { and } u \in \partial P\left(\theta, \rho_{2}\right)
$$

and

(i) $\Phi(A u)>\rho_{3}$ for all $u \in \partial P\left(\Phi, \rho_{3}\right)$;

(ii) $\theta(A u)<\rho_{2}$ for all $u \in \partial P\left(\theta, \rho_{2}\right)$;

(iii) $P\left(v, \rho_{1}\right) \neq \emptyset$ and $v(A u)>\rho_{1}$ for all $u \in \partial P\left(v, \rho_{1}\right)$.

Then $A$ has at least two fixed points $u_{1}$ and $u_{2}$ belonging to $\overline{P\left(\Phi, \rho_{3}\right)}$ satisfying

$$
\rho_{1}<v\left(u_{1}\right) \quad \text { with } \theta\left(u_{1}\right)<\rho_{2} \text {, and } \rho_{2}<\theta\left(u_{2}\right) \quad \text { with } \Phi\left(u_{2}\right)<\rho_{3} \text {. }
$$

Define a constant $l \in(0,1)_{\mathbb{T}}$ such that $0<\xi_{m-2}<l<1$. Let $\Phi, \theta$ and $v$ be increasing, nonnegative and continuous functionals on $P$, defined by

$$
\Phi(u)=u\left(\xi_{m-2}\right), \quad \theta(u)=u\left(\xi_{m-2}\right), \quad v(u)=u(l) .
$$

Obviously, $\Phi(u)=\theta(u) \leq v(u)$ for each $u \in P$. Moreover, Lemma 2.5 implies $\Phi(u)=$ $u\left(\xi_{m-2}\right) \geq \gamma\|u\|$ for each $u \in P$. It is easy to see that $\theta(0)=0$ and $\theta(\lambda u)=\lambda \theta(u)$ for all $0 \leq \lambda \leq 1$ and $u \in \partial P\left(\theta, \rho_{2}\right)$.

We can now prove the following theorem.

Theorem 4.2 Assume that $\left(\mathrm{H}_{1}\right)-\left(\mathrm{H}_{3}\right)$ hold, and suppose that there exist positive numbers $\rho_{1}<\rho_{2}<\rho_{3}$ such that the function $f$ satisfies the following conditions:

(B $\left.\mathrm{B}_{1}\right) f(t, u)>\varphi_{p}(N \gamma) \varphi_{p}\left(\rho_{1}\right)$ for $t \in\left[\xi_{m-2}, l\right]_{\mathbb{T}}$ and $u \in\left[\gamma \rho_{1}, \rho_{1}\right]$;

$\left(\mathrm{B}_{2}\right) f(t, u)<\varphi_{p}(L) \varphi_{p}\left(\rho_{2}\right)$ for $t \in\left[\xi_{m-2}, 1\right]_{\mathbb{T}}$ and $u \in\left[0, \rho_{2}\right]$;

(B $\left.\mathrm{B}_{3}\right) f(t, u)>\varphi_{p}(M \gamma) \varphi_{p}\left(\rho_{3}\right)$ for $t \in\left[\xi_{m-2}, l\right]_{\mathbb{T}}$ and $u \in\left[\rho_{3},(1 / \gamma) \rho_{3}\right]$,

where constants $L, M, N$ are defined by (2.12), (2.13) and (2.14), respectively.

Then problem (1.7)-(1.8) has at least two positive solutions $u_{1}$ and $u_{2}$ such that $\rho_{1}<u_{1}(l)$ with $u_{1}\left(\xi_{m-2}\right)<\rho_{2}$ and $\rho_{2}<u_{2}\left(\xi_{m-2}\right)$ with $u_{2}\left(\xi_{m-2}\right)<\rho_{3}$.

Proof We now wish to prove that all of the conditions of Theorem 4.1 are satisfied. For this purpose, we define the cone $P$ as (2.7) and a completely continuous operator $A: P \rightarrow P$ by (2.8).

To check condition (i) of Theorem 4.1, we choose $u \in \partial P\left(\Phi, \rho_{3}\right)$, then $\Phi(u)=\rho_{3}$. This implies that $\rho_{3} \leq\|u\| \leq(1 / \gamma) \Phi(u)=(1 / \gamma) \rho_{3}$. For $t \in\left[\xi_{m-2}, 1\right]_{\mathbb{T}}$, we have $\rho_{3} \leq u(t) \leq(1 / \gamma) \rho_{3}$. From condition $\left(\mathrm{B}_{3}\right)$, we get that $f(t, u)>\varphi_{p}(M \gamma) \varphi_{p}\left(\rho_{3}\right)$ for $t \in\left[\xi_{m-2}, l\right]_{\mathbb{T}}$. Since $A u \in P$, we obtain

$$
\begin{aligned}
\Phi(A u)= & (A u)\left(\xi_{m-2}\right) \\
= & -\int_{0}^{\xi_{m-2}} \varphi_{q}\left(\int_{0}^{\tau} a(s) f(s, u(s)) \Delta s\right) \Delta \tau \\
& -\frac{1}{1-\Lambda} \sum_{i=1}^{m-1} \alpha_{i} \int_{\xi_{i-1}}^{\xi_{i}} \int_{0}^{\eta} \varphi_{q}\left(\int_{0}^{\tau} a(s) f(s, u(s)) \Delta s\right) \Delta \tau \Delta \eta
\end{aligned}
$$




$$
\begin{aligned}
& +\frac{1}{1-\Lambda} \int_{0}^{1} \varphi_{q}\left(\int_{0}^{\tau} a(s) f(s, u(s)) \Delta s\right) \Delta \tau \\
& =\frac{\int_{0}^{1} \varphi_{q}\left(\int_{0}^{\tau} a(s) f(s, u(s)) \Delta s\right) \Delta \tau-\int_{0}^{\xi_{m-2}} \varphi_{q}\left(\int_{0}^{\tau} a(s) f(s, u(s)) \Delta s\right) \Delta \tau}{1-\Lambda} \\
& +\frac{1}{1-\Lambda}\left[\Lambda \int_{0}^{\xi_{m-2}} \varphi_{q}\left(\int_{0}^{\tau} a(s) f(s, u(s)) \Delta s\right) \Delta \tau\right. \\
& \left.-\sum_{i=1}^{m-1} \alpha_{i} \int_{\xi_{i-1}}^{\xi_{i}} \int_{0}^{\eta} \varphi_{q}\left(\int_{0}^{\tau} a(s) f(s, u(s)) \Delta s\right) \Delta \tau \Delta \eta\right] \\
& \geq \frac{1}{1-\Lambda} \int_{\xi_{m-2}}^{1} \varphi_{q}\left(\int_{0}^{\tau} a(s) f(s, u(s)) \Delta s\right) \Delta \tau \\
& -\frac{1}{1-\Lambda} \int_{\xi_{m-2}}^{1} \int_{\xi_{m-2}}^{\eta} \varphi_{q}\left(\int_{0}^{\tau} a(s) f(s, u(s)) \Delta s\right) \Delta \tau \Delta \eta \\
& \geq \frac{1}{1-\Lambda} \int_{\xi_{m-2}}^{1} \varphi_{q}\left(\int_{0}^{\tau} a(s) f(s, u(s)) \Delta s\right) \Delta \tau \\
& -\frac{1}{1-\Lambda} \int_{\xi_{m-2}}^{1} \int_{\xi_{m-2}}^{1} \varphi_{q}\left(\int_{0}^{\tau} a(s) f(s, u(s)) \Delta s\right) \Delta \tau \Delta \eta \\
& =\frac{\xi_{m-2}}{1-\Lambda} \int_{\xi_{m-2}}^{1} \varphi_{q}\left(\int_{0}^{\tau} a(s) f(s, u(s)) \Delta s\right) \Delta \tau \\
& \geq \frac{\xi_{m-2}}{1-\Lambda} \int_{\xi_{m-2}}^{1} \varphi_{q}\left(\int_{\xi_{m-2}}^{\tau} a(s) f(s, u(s)) \Delta s\right) \Delta \tau \\
& >\varphi_{q}\left(\varphi_{p}(M \gamma) \varphi_{p}\left(\rho_{3}\right)\right) \frac{\xi_{m-2} \int_{\xi_{m-2}}^{1} \varphi_{q}\left(\int_{\xi_{m-2}}^{\tau} a(s) \Delta s\right) \Delta \tau}{1-\Lambda} \\
& =\frac{M \gamma \rho_{3}}{1-\Lambda} \xi_{m-2} \int_{\xi_{m-2}}^{1} \varphi_{q}\left(\int_{\xi_{m-2}}^{\tau} a(s) \Delta s\right) \Delta \tau \\
& =\rho_{3} \text {. }
\end{aligned}
$$

Hence, condition (i) of Theorem 4.1 holds.

We now prove that condition (ii) in Theorem 4.1 holds. In fact, for $u \in \partial P\left(\theta, \rho_{2}\right)$, we have $\theta(u)=\rho_{2}$. This implies that $0 \leq u(t) \leq\|u\| \leq(1 / \gamma) \rho_{2}$ for $t \in\left[\xi_{m-2}, 1\right]_{\mathbb{T}}$. From condition $\left(\mathrm{B}_{2}\right)$, we have

$$
\begin{aligned}
\theta(A u) & =(A u)\left(\xi_{m-2}\right) \\
& \leq \frac{1}{1-\Lambda} \int_{0}^{1} \varphi_{q}\left(\int_{0}^{\tau} a(s) f(s, u(s)) \Delta s\right) \Delta \tau \\
& <\frac{\varphi_{q}\left(\varphi_{p}(L) \varphi_{p}\left(\rho_{2}\right)\right)}{1-\Lambda} \int_{0}^{1} \varphi_{q}\left(\int_{0}^{\tau} a(s) \Delta s\right) \Delta \tau \\
& =\frac{L \rho_{2}}{1-\Lambda} \int_{0}^{1} \varphi_{q}\left(\int_{0}^{\tau} a(s) \Delta s\right) \Delta \tau \\
& =\rho_{2}=\|u\| .
\end{aligned}
$$

This shows that condition (ii) of Theorem 4.1 is satisfied. 
Now, we assert that condition (iii) of Theorem 4.1 also holds. If $u(t)=\rho_{1} / 2$ for $t \in[0,1]_{\mathbb{T}}$, then $v(u)=\rho_{1} / 2$. Thus $P\left(v, \rho_{1}\right) \neq \emptyset$. Let $u \in \partial P\left(v, \rho_{1}\right)$, then $v(u)=u(l)=\rho_{1}$. So that $\gamma \rho_{1} \leq$ $u(t) \leq\|u\| \leq \rho_{1}$. From condition $\left(\mathrm{B}_{1}\right)$, for any $A u \in P$, we have

$$
\begin{aligned}
& v(A u)=(A u)(l) \geq(A u)(1) \\
& =-\int_{0}^{1} \varphi_{q}\left(\int_{0}^{\tau} a(s) f(s, u(s)) \Delta s\right) \Delta \tau \\
& -\frac{1}{1-\Lambda} \sum_{i=1}^{m-1} \alpha_{i} \int_{\xi_{i-1}}^{\xi_{i}} \int_{0}^{\eta} \varphi_{q}\left(\int_{0}^{\tau} a(s) f(s, u(s)) \Delta s\right) \Delta \tau \Delta \eta \\
& +\frac{1}{1-\Lambda} \int_{0}^{1} \varphi_{q}\left(\int_{0}^{\tau} a(s) f(s, u(s)) \Delta s\right) \Delta \tau \\
& =\frac{1}{1-\Lambda}\left[\Lambda \int_{0}^{1} \varphi_{q}\left(\int_{0}^{\tau} a(s) f(s, u(s)) \Delta s\right) \Delta \tau\right. \\
& \left.-\sum_{i=1}^{m-1} \alpha_{i} \int_{\xi_{i-1}}^{\xi_{i}} \int_{0}^{\eta} \varphi_{q}\left(\int_{0}^{\tau} a(s) f(s, u(s)) \Delta s\right) \Delta \tau \Delta \eta\right] \\
& =\frac{1}{1-\Lambda}\left[\sum_{i=1}^{m-1} \alpha_{i} \int_{\xi_{i-1}}^{\xi_{i}} \int_{0}^{1} \varphi_{q}\left(\int_{0}^{\tau} a(s) f(s, u(s)) \Delta s\right) \Delta \tau \Delta \eta\right. \\
& \left.-\sum_{i=1}^{m-1} \alpha_{i} \int_{\xi_{i-1}}^{\xi_{i}} \int_{0}^{\eta} \varphi_{q}\left(\int_{0}^{\tau} a(s) f(s, u(s)) \Delta s\right) \Delta \tau \Delta \eta\right] \\
& =\frac{1}{1-\Lambda} \sum_{i=1}^{m-1} \alpha_{i} \int_{\xi_{i-1}}^{\xi_{i}} \int_{\eta}^{1} \varphi_{q}\left(\int_{0}^{\tau} a(s) f(s, u(s)) \Delta s\right) \Delta \tau \Delta \eta \\
& \geq \frac{1}{1-\Lambda} \sum_{i=1}^{m-1} \alpha_{i} \int_{\xi_{i-1}}^{\xi_{i}} \int_{\xi_{m-2}}^{1} \varphi_{q}\left(\int_{0}^{\tau} a(s) f(s, u(s)) \Delta s\right) \Delta \tau \Delta \eta \\
& \geq \frac{\Lambda}{1-\Lambda} \int_{\xi_{m-2}}^{1} \varphi_{q}\left(\int_{\xi_{m-2}}^{\tau} a(s) f(s, u(s)) \Delta s\right) \Delta \tau \\
& >\varphi_{q}\left(\varphi_{p}(N \gamma) \varphi_{p}\left(\rho_{1}\right)\right) \frac{\Lambda \int_{\xi_{m-2}}^{1} \varphi_{q}\left(\int_{\xi_{m-2}}^{\tau} a(s) \Delta s\right) \Delta \tau}{1-\Lambda} \\
& =\frac{N \gamma \rho_{1} \Lambda}{1-\Lambda} \int_{\xi_{m-2}}^{1} \varphi_{q}\left(\int_{\xi_{m-2}}^{\tau} a(s) \Delta s\right) \Delta \tau=\rho_{1} .
\end{aligned}
$$

Therefore, condition (iii) of Theorem 4.1 is satisfied.

Thus, by Theorem 4.1, problem (1.7)-(1.8) has at least two positive solutions $u_{1}$ and $u_{2}$ such that $\rho_{1}<u_{1}(l)$ with $u_{1}\left(\xi_{m-2}\right)<\rho_{2}$ and $\rho_{2}<u_{2}\left(\xi_{m-2}\right)$ with $u_{2}\left(\xi_{m-2}\right)<\rho_{3}$.

\section{Existence of at least three positive solutions}

In this section, we use the Leggett-Williams fixed point theorem to prove the existence of at least three positive solutions to problem (1.7)-(1.8). The Leggett-Williams fixed point theorem is as follows. 
Theorem 5.1 (see [30]) Let P be a cone in the real Banach space E. Set

$$
P_{r}=\{x \in P \mid\|x\|<r\}, \quad P(\Psi, a, b)=\{x \in P \mid a \leq \Psi(x),\|x\| \leq b\} .
$$

Let $A: \bar{P}_{r} \rightarrow \bar{P}_{r}$ be a completely continuous operator and let $\Psi$ be a nonnegative continuous concave functional on $P$ with $\Psi(u) \leq\|u\|$ for all $u \in \bar{P}_{r}$. Suppose that there exists $0<\rho_{1}<$ $\rho_{2}<(1 / \gamma) \rho_{2}<\rho_{3}$ such that the following conditions hold:

(i) $\left\{u \in P\left(\Psi, \rho_{2},(1 / \gamma) \rho_{2}\right) \mid \Psi(u)>\rho_{2}\right\} \neq \emptyset$ and $\Psi(A u)>\rho_{2}$ for all $u \in \partial P\left(\Psi, \rho_{2},(1 / \gamma) \rho_{2}\right)$;

(ii) $\|A u\|<\rho_{1}$ for $\|u\| \leq \rho_{1}$

(iii) $\Psi(A u)>\rho_{2}$ for $u \in P\left(\Psi, \rho_{2}, \rho_{3}\right)$ with $\|A u\|>(1 / \gamma) \rho_{2}$.

Then $A$ has at least three fixed points $u_{1}, u_{2}$ and $u_{3}$ in $\bar{P}_{r}$ satisfying $\left\|u_{1}\right\|<\rho_{1}, \Psi\left(u_{2}\right)>\rho_{2}$, $\rho_{1}<\left\|u_{3}\right\|$ with $\Psi\left(u_{3}\right)<\rho_{2}$.

We now prove the following result.

Theorem 5.2 Assume that $\left(\mathrm{H}_{1}\right)-\left(\mathrm{H}_{3}\right)$ hold. Suppose that there exist constants $0<\rho_{1}<\rho_{2}<$ $(1 / \gamma) \rho_{2} \leq \rho_{3}$ such that

$\left(C_{1}\right) f(t, u) \leq \varphi_{p}(L) \varphi_{p}\left(\rho_{3}\right)$ for $t \in\left[\xi_{m-2}, 1\right]_{\mathbb{T}}$ and $u \in\left[0, \rho_{3}\right]$;

$\left(C_{2}\right) f(t, u)>\varphi_{p}(M \gamma) \varphi_{p}\left(\rho_{2}\right)$ for $t \in\left[\xi_{m-2}, 1\right]_{\mathbb{T}}$ and $u \in\left[\rho_{2},(1 / \gamma) \rho_{2}\right]$;

$\left(C_{3}\right) f(t, u)<\varphi_{p}(L) \varphi_{p}\left(\rho_{1}\right)$ for $t \in\left[\xi_{m-2}, 1\right]_{\mathbb{T}}$ and $u \in\left[0, \rho_{1}\right]$,

where constants $L, M$ are defined by (2.12) and (2.13), respectively.

Then problem (1.7)-(1.8) has at least three positive solutions $u_{1}, u_{2}$ and $u_{3}$ such that $\left\|u_{1}\right\|<$ $\rho_{1}, u_{2}\left(\xi_{m-2}\right)>\rho_{2},\left\|u_{3}\right\|>\rho_{1}$ with $u_{3}\left(\xi_{m-2}\right)<\rho_{2}$.

Proof We will show that all the conditions of Leggett-Williams Theorem 5.1 hold with respect to the operator $A$ defined in (2.8).

At first, we define a nonnegative continuous concave functional $\Psi: P \rightarrow[0, \infty)$ by $\Psi(u)=u\left(\xi_{m-2}\right)$, where the cone $P$ is defined by (2.7). In fact, for $u \in P$, we get $\Psi(u) \leq\|u\|$. If $u \in \bar{P}_{\rho_{3}}$, then $\|u\| \leq \rho_{3}$. From condition $\left(\mathrm{C}_{1}\right)$, we obtain

$$
\begin{aligned}
A u(t)= & -\int_{0}^{t} \varphi_{q}\left(\int_{0}^{\tau} a(s) f(s, u(s)) \Delta s\right) \Delta \tau \\
& -\frac{1}{1-\Lambda} \sum_{i=1}^{m-1} \alpha_{i} \int_{\xi_{i-1}}^{\xi_{i}} \int_{0}^{\eta} \varphi_{q}\left(\int_{0}^{\tau} a(s) f(s, u(s)) \Delta s\right) \Delta \tau \Delta \eta \\
& +\frac{1}{1-\Lambda} \int_{0}^{1} \varphi_{q}\left(\int_{0}^{\tau} a(s) f(s, u(s)) \Delta s\right) \Delta \tau \\
\leq & \frac{1}{1-\Lambda} \int_{0}^{1} \varphi_{q}\left(\int_{0}^{\tau} a(s) f(s, u(s)) \Delta s\right) \Delta \tau \\
\leq & \frac{\varphi_{q}\left(\varphi_{p}(L) \varphi_{p}\left(\rho_{3}\right)\right)}{1-\Lambda} \int_{0}^{1} \varphi_{q}\left(\int_{0}^{\tau} a(s) \Delta s\right) \Delta \tau \\
= & \frac{L \rho_{3}}{1-\Lambda} \int_{0}^{1} \varphi_{q}\left(\int_{0}^{\tau} a(s) \Delta s\right) \Delta \tau=\rho_{3} .
\end{aligned}
$$

This implies that $\|A u\| \leq \rho_{3}$. Therefore, we have $A: \bar{P}_{\rho_{3}} \rightarrow \bar{P}_{\rho_{3}}$. Since $\left(\rho_{2} / \gamma\right) \in$ $P\left(\Psi, \rho_{2},\left(\rho_{2} / \gamma\right)\right)$ and $\Psi\left(\left(\rho_{2} / \gamma\right)\right)=\left(\rho_{2} / \gamma\right)>\rho_{2}$, then $\left\{u \in P\left(\Psi, \rho_{2},\left(\rho_{2} / \gamma\right)\right) \mid \Psi(u)>\rho_{2}\right\} \neq \emptyset$. 
For $u \in P\left(\Psi, \rho_{2},\left(\rho_{2} / \gamma\right)\right)$, we get $\rho_{2} \leq u\left(\xi_{m-2}\right) \leq\|u\| \leq\left(\rho_{2} / \gamma\right)$. By using condition $\left(C_{2}\right)$, we obtain

$$
\begin{aligned}
& \Psi(A u)=(A u)\left(\xi_{m-2}\right) \\
& =-\int_{0}^{\xi_{m-2}} \varphi_{q}\left(\int_{0}^{\tau} a(s) f(s, u(s)) \Delta s\right) \Delta \tau \\
& -\frac{1}{1-\Lambda} \sum_{i=1}^{m-1} \alpha_{i} \int_{\xi_{i-1}}^{\xi_{i}} \int_{0}^{\eta} \varphi_{q}\left(\int_{0}^{\tau} a(s) f(s, u(s)) \Delta s\right) \Delta \tau \Delta \eta \\
& +\frac{1}{1-\Lambda} \int_{0}^{1} \varphi_{q}\left(\int_{0}^{\tau} a(s) f(s, u(s)) \Delta s\right) \Delta \tau \\
& =\frac{\int_{0}^{1} \varphi_{q}\left(\int_{0}^{\tau} a(s) f(s, u(s)) \Delta s\right) \Delta \tau-\int_{0}^{\xi_{m-2}} \varphi_{q}\left(\int_{0}^{\tau} a(s) f(s, u(s)) \Delta s\right) \Delta \tau}{1-\Lambda} \\
& +\frac{1}{1-\Lambda}\left[\Lambda \int_{0}^{\xi_{m-2}} \varphi_{q}\left(\int_{0}^{\tau} a(s) f(s, u(s)) \Delta s\right) \Delta \tau\right. \\
& \left.-\sum_{i=1}^{m-1} \alpha_{i} \int_{\xi_{i-1}}^{\xi_{i}} \int_{0}^{\eta} \varphi_{q}\left(\int_{0}^{\tau} a(s) f(s, u(s)) \Delta s\right) \Delta \tau \Delta \eta\right] \\
& \geq \frac{1}{1-\Lambda} \int_{\xi m-2}^{1} \varphi_{q}\left(\int_{0}^{\tau} a(s) f(s, u(s)) \Delta s\right) \Delta \tau \\
& -\frac{1}{1-\Lambda} \int_{\xi_{m-2}}^{1} \int_{\xi_{m-2}}^{\eta} \varphi_{q}\left(\int_{0}^{\tau} a(s) f(s, u(s)) \Delta s\right) \Delta \tau \Delta \eta \\
& \geq \frac{1}{1-\Lambda} \int_{\xi_{m-2}}^{1} \varphi_{q}\left(\int_{0}^{\tau} a(s) f(s, u(s)) \Delta s\right) \Delta \tau \\
& -\frac{1}{1-\Lambda} \int_{\xi_{m-2}}^{1} \int_{\xi_{m-2}}^{1} \varphi_{q}\left(\int_{0}^{\tau} a(s) f(s, u(s)) \Delta s\right) \Delta \tau \Delta \eta \\
& =\frac{\xi_{m-2}}{1-\Lambda} \int_{\xi_{m-2}}^{1} \varphi_{q}\left(\int_{0}^{\tau} a(s) f(s, u(s)) \Delta s\right) \Delta \tau \\
& \geq \frac{\xi_{m-2}}{1-\Lambda} \int_{\xi_{m-2}}^{1} \varphi_{q}\left(\int_{\xi_{m-2}}^{\tau} a(s) f(s, u(s)) \Delta s\right) \Delta \tau \\
& >\varphi_{q}\left(\varphi_{p}(M \gamma) \varphi_{p}\left(\rho_{2}\right)\right) \frac{\xi_{m-2} \int_{\xi_{m-2}}^{1} \varphi_{q}\left(\int_{\xi_{m-2}}^{\tau} a(s) \Delta s\right) \Delta \tau}{1-\Lambda} \\
& =\frac{M \gamma \rho_{2}}{1-\Lambda} \xi_{m-2} \int_{\xi_{m-2}}^{1} \varphi_{q}\left(\int_{\xi_{m-2}}^{\tau} a(s) \Delta s\right) \Delta \tau=\rho_{2} .
\end{aligned}
$$

Hence, condition (i) of Theorem 5.1 is satisfied.

Indeed, if $\|u\| \leq \rho_{1}$, then condition $\left(\mathrm{C}_{3}\right)$ implies that

$$
\begin{aligned}
(A u)(t) & <\frac{\varphi_{q}\left(\varphi_{p}(L) \varphi_{p}\left(\rho_{1}\right)\right)}{1-\Lambda} \int_{0}^{1} \varphi_{q}\left(\int_{0}^{\tau} a(s) \Delta s\right) \Delta \tau \\
& =\frac{L \rho_{1}}{1-\Lambda} \int_{0}^{1} \varphi_{q}\left(\int_{0}^{\tau} a(s) \Delta s\right) \Delta \tau=\rho_{1} .
\end{aligned}
$$

Thus $\|A u\|<\rho_{1}$. Therefore, condition (ii) of Theorem 5.1 holds. 
We finally show that condition (iii) of Theorem 5.1 also holds. Assume that $u \in$ $P\left(\Psi, \rho_{2}, \rho_{3}\right)$, with $\|A u\|>(1 / \gamma) \rho_{2}$. Then we obtain

$$
\begin{aligned}
\Psi(A u) & =(A u)\left(\xi_{m-2}\right) \\
& \geq(A u)(1) \\
& \geq \gamma\|A u\|>\rho_{2} .
\end{aligned}
$$

So, condition (iii) of Theorem 5.1 is satisfied. Therefore, an application of Theorem 5.1 implies that problem (1.7)-(1.8) has at least three positive solutions $u_{1}, u_{2}$ and $u_{3}$ such that $\left\|u_{1}\right\|<\rho_{1}, u_{2}\left(\xi_{m-2}\right)>\rho_{2}$ and $\left\|u_{3}\right\|>\rho_{1}$ with $u_{3}\left(\xi_{m-2}\right)<\rho_{2}$.

\section{Numerical examples}

In this section, we present some examples to illustrate our results.

Example 6.1 Consider the following six-point integral boundary value problem with $p=$ 3 and $\mathbb{T}=\mathbb{R}$ :

$$
\begin{aligned}
& \left(\varphi_{p}\left(u^{\Delta}(t)\right)\right)^{\Delta}+f(t, u(t))=0, \quad t \in[0,1]_{\mathbb{T}}, \\
& u^{\Delta}(0)=0, \quad u(1)=\frac{1}{4} \int_{0}^{1 / 5} u(s) \Delta s+\frac{1}{5} \int_{2 / 5}^{3 / 5} u(s) \Delta s+2 \int_{3 / 5}^{4 / 5} u(s) \Delta s,
\end{aligned}
$$

where

$$
f(t, u)= \begin{cases}\frac{1}{100} t+u^{3}, & t \in[0,1], u \in\left[0, \frac{1}{5}\right], \\ \frac{1}{100} t+u^{3}+100\left(u-\frac{1}{5}\right)^{1 / 4}, & t \in[0,1], u \in\left[\frac{1}{5}, \frac{3}{5}\right] \\ \frac{1}{100} t+u^{3}+100\left(u-\frac{1}{5}\right)^{1 / 4}+10\left(u-\frac{3}{5}\right), & t \in[0,1], u \in\left[\frac{3}{5}, \infty\right)\end{cases}
$$

Set $\alpha_{1}=1 / 4, \alpha_{3}=1 / 5, \alpha_{4}=2, \alpha_{2}=\alpha_{5}=0, \xi_{0}=0, \xi_{1}=1 / 5, \xi_{2}=2 / 5, \xi_{3}=3 / 5, \xi_{4}=4 / 5, \xi_{5}=1$ and $a(t)=1$. We can show that

$$
\Lambda=\sum_{i=1}^{5} \alpha_{i}\left(\xi_{i}-\xi_{i-1}\right)=\frac{49}{100}<1 .
$$

Through a simple calculation we can get

$$
\begin{aligned}
& \gamma=\frac{\alpha_{m-2}\left(\xi_{m-2}-\xi_{m-3}\right)\left(1-\xi_{m-2}\right)}{1-\alpha_{m-2} \xi_{m-2}\left(\xi_{m-2}-\xi_{m-3}\right)}=\frac{2}{17}, \\
& M=\frac{1-\Lambda}{\gamma \xi_{m-2} \int_{\xi_{m-2}}^{1} \varphi_{q}\left(\int_{\xi_{m-2}}^{\tau} a(s) \Delta s\right) \Delta \tau}=\frac{2601}{64} \sqrt{5}, \\
& L=\frac{1-\Lambda}{\int_{0}^{1} \varphi_{q}\left(\int_{0}^{\tau} a(s) \Delta s\right) \Delta \tau}=\frac{153}{200} .
\end{aligned}
$$

Choose $r=1 / 5$ and $R=3 / 5$, then $f(t, u)$ satisfies

$$
f(t, u) \leq \frac{1}{100}+\left(\frac{1}{5}\right)^{3}<\left(\frac{153}{200} \times \frac{1}{5}\right)^{2}=\varphi_{3}(L r), \quad t \in[0,1], u \in\left[0, \frac{1}{5}\right],
$$


and

$$
\begin{aligned}
f(t, u) & \geq \frac{1}{100}\left(\frac{4}{5}\right)+\left(\frac{3}{5}\right)^{3}+100\left(\frac{3}{5}-\frac{1}{5}\right)^{1 / 4} \\
& >\left(\frac{2601 \sqrt{5}}{64} \times \frac{2}{17} \times \frac{3}{5}\right)^{2}=\varphi_{3}(M \gamma R), \quad t \in\left[\frac{4}{5}, 1\right], u \in\left[\frac{3}{5}, \infty\right) .
\end{aligned}
$$

By Theorem 3.2, we have that boundary value problem (6.1)-(6.2) has at least one positive solution.

Example 6.2 Consider the following six-point integral boundary value problem with $p=$ 2 and $\mathbb{T}=\{0\} \cup\left\{1 / 2^{n}: n \in \mathbb{N}\right\} \cup\left(\frac{1}{2}, 1\right]$ (N stands for the natural number set).

$$
\begin{aligned}
& \left(\varphi_{p}\left(u^{\Delta}(t)\right)\right)^{\Delta}+f(t, u(t))=0, \quad t \in[0,1]_{\mathbb{T}}, \\
& u^{\Delta}(0)=0, \quad u(1)=\frac{1}{4} \int_{0}^{1 / 16} u(s) \Delta s+\frac{1}{6} \int_{1 / 8}^{1 / 4} u(s) \Delta s+3 \int_{1 / 4}^{1 / 2} u(s) \Delta s,
\end{aligned}
$$

where

$$
f(t, u)=\left\{\begin{aligned}
\frac{1}{50} t & +\frac{1}{100} u \\
& t \in\left[\frac{1}{2}, 1\right], u \in[0,1], \\
\frac{1}{50} t & +\frac{1}{100} u+7(u-1)^{1 / 6} \\
& t \in\left[\frac{1}{2}, 1\right], u \in[1,2], \\
\frac{1}{50} t & +\frac{1}{100} u+7(u-1)^{1 / 6}+\frac{1}{20}(u-2)^{1 / 2} \\
& t \in\left[\frac{1}{2}, 1\right], u \in\left[2, \frac{4096}{585}\right], \\
\frac{1}{50} t & +\frac{1}{100} u+7(u-1)^{1 / 6}+\frac{1}{20}(u-2)^{1 / 2}+\frac{1}{40}\left(u-\frac{4096}{585}\right), \\
& t \in\left[\frac{1}{2}, 1\right], u \in\left[\frac{4096}{585}, 30\right] .
\end{aligned}\right.
$$

Set $\alpha_{1}=1 / 4, \alpha_{3}=1 / 6, \alpha_{4}=3, \alpha_{2}=\alpha_{5}=0, \xi_{0}=0, \xi_{1}=1 / 16, \xi_{2}=1 / 8, \xi_{3}=1 / 4, \xi_{4}=1 / 2, \xi_{5}=1$ and $a(t)=1$. We can show that

$$
\Lambda=\sum_{i=1}^{5} \alpha_{i}\left(\xi_{i}-\xi_{i-1}\right)=\frac{151}{192}<1 .
$$

Through a simple calculation we can get

$$
\begin{aligned}
& \gamma=\frac{\alpha_{m-2}\left(\xi_{m-2}-\xi_{m-3}\right)\left(1-\xi_{m-2}\right)}{1-\alpha_{m-2} \xi_{m-2}\left(\xi_{m-2}-\xi_{m-3}\right)}=\frac{3}{5}, \\
& M=\frac{1-\Lambda}{\gamma \xi_{m-2} \int_{\xi_{m-2}}^{1} \varphi_{q}\left(\int_{\xi_{m-2}}^{\tau} a(s) \Delta s\right) \Delta \tau}=\frac{205}{36}, \\
& L=\frac{1-\Lambda}{\int_{0}^{1} \varphi_{q}\left(\int_{0}^{\tau} a(s) \Delta s\right) \Delta \tau}=\frac{41}{88} .
\end{aligned}
$$

Choose $\rho_{1}=1, \rho_{2}=2$ and $\rho_{3}=30$, then $f(t, u)$ satisfies

$$
f(t, u) \leq \frac{1}{50}+\frac{1}{100}<\frac{41}{88} \times 1=\varphi_{2}\left(L \rho_{1}\right), \quad t \in\left[\frac{1}{2}, 1\right], u \in[0,1]
$$


and

$$
\begin{aligned}
f(t, u) & \geq \frac{1}{50}\left(\frac{1}{2}\right)+\frac{1}{100}(2)+7(2-1)^{1 / 6} \\
& >\frac{205}{36} \times \frac{3}{5} \times 2=\varphi_{2}\left(M \gamma \rho_{2}\right), \quad t \in\left[\frac{1}{2}, 1\right], u \in\left[2, \frac{4096}{585}\right],
\end{aligned}
$$

and

$$
\begin{aligned}
f(t, u) & \leq \frac{1}{50}+\frac{1}{100}(30)+7(30-1)^{1 / 6}+\frac{1}{20}(30-2)^{1 / 2}+\frac{1}{40}\left(30-\frac{4096}{585}\right) \\
& <\frac{41}{88} \times 30=\varphi_{2}\left(L \rho_{3}\right), \quad t \in\left[\frac{1}{2}, 1\right], u \in[0,30] .
\end{aligned}
$$

By Theorem 5.2, we get that problem (6.3)-(6.4) has at least three positive solutions $u_{1}, u_{2}$ and $u_{3}$ such that $\left\|u_{1}\right\|<1, u_{2}\left(\frac{1}{2}\right)>2$ and $\left\|u_{3}\right\|>1$ with $u_{3}\left(\frac{1}{2}\right)<2$.

\section{Competing interests}

The authors declare that they have no competing interests.

\section{Authors' contributions}

Both authors contributed equally in this article. They read and approved the final manuscript.

\section{Acknowledgements}

We would like to thank the reviewers for their valuable comments and suggestions on the manuscript. This research is supported by King Mongkut's University of Technology North Bangkok, Thailand.

\section{Received: 17 February 2013 Accepted: 27 June 2013 Published: 11 July 2013}

\section{References}

1. Hilger, S: Analysis on measure chains - a unified approach to continuous and discrete calculus. Results Math. 18, 18-56 (1990)

2. Agarwal, RP, Bohner, M: Basic calculus on time scales and some of its applications. Results Math. 35, 3-22 (1999)

3. Agarwal, RP, Bohner, M, O'Regan, D, Peterson, A: Dynamic equations on time scales: a survey. J. Comput. Appl. Math. $141,1-26(2002)$

4. Bohner, M, Peterson, A: Dynamic Equations on Time Scales: An Introduction with Applications. Birkhäuser, Boston (2001)

5. Bohner, M, Peterson, A: Advances in Dynamic Equations on Time Scales. Birkhäuser, Boston (2003)

6. Lakshmikantham, V, Sivasundaram, S, Kaymakcalan, B: Dynamic Systems on Measure Chains. Kluwer Academic, Boston (1996)

7. Atici, FM, Biles, DC, Lebedinsky, A: An application of time scales to economics. Math. Comput. Model. 43, 718-726 (2006)

8. Jones, MA, Song, B, Thomas, DM: Controlling wound healing through debridement. Math. Comput. Model. 40, 1057-1064 (2004)

9. Spedding, V: Taming nature's numbers. New Sci. 179, 28-32 (2003)

10. Thomas, DM, Vandemuelebroeke, L, Yamaguchi, K: A mathematical evolution model for phytoremediation of metals. Discrete Contin. Dyn. Syst., Ser. B 5, 411-422 (2005)

11. Gallardo, JM: Second order differential operators with integral boundary conditions and generation of semigroups. Rocky Mt. J. Math. 30, 1265-1292 (2000)

12. Karakostas, GL, Tsamatos, PCh: Multiple positive solutions of some Fredholm integral equations arisen from nonlocal boundary-value problems. Electron. J. Differ. Equ. 30, 1-17 (2002)

13. Lomtatidze, A, Malaguti, L: On a nonlocal boundary-value problems for second order nonlinear singular differential equations. Georgian Math. J. 7, 133-154 (2000)

14. Corduneanu, C: Integral Equations and Applications. Cambridge University Press, Cambridge (1991)

15. Agarwal, RP, O’Regan, D: Infinite Interval Problems for Differential, Difference and Integral Equations. Kluwer Academic, Dordrecht (2001)

16. Sun, HR, Li, WT: Existence theory for positive solutions to one-dimensional $p$-Laplacian boundary value problems on time scales. J. Differ. Equ. 240, 217-248 (2007)

17. Zhang, Y, Qiao, S: Existence of positive solutions for $m$-point boundary value problems on time scales. Discrete Dyn. Nat. Soc. 2009, Article ID 189768 (2009)

18. Li, Y, Zhang, T: Multiple positive solutions for second-order $p$-Laplacian dynamic equations with integral boundary conditions. Bound. Value Probl. 2011, Article ID 867615 (2011) 
19. Su, YH: Arbitrary positive solutions to a multi-point $p$-Laplacian boundary value problem involving the derivative on time scales. Math. Comput. Model. 53, 1742-1747 (2011)

20. Goodrich, CS: Existence of a positive solution to a first-order $p$-Laplacian BVP on a time scale. Nonlinear Anal. 74, 1926-1936 (2011)

21. Song, C, Gao, X: Positive solutions for third-order p-Laplacian functional dynamic equations on time scales. Bound. Value Probl. 2011, Article ID 279752 (2011)

22. Su, Y, Feng, Z: Positive solutions to the singular $p$-Laplacian BVPs with sign-changing nonlinearities and higher-order derivatives in Banach spaces on time scales. Dyn. Partial Differ. Equ. 8, 149-171 (2011)

23. Su, H, Liu, L, Wang, X: Solutions for $p$-Laplacian dynamic delay differential equations on time scales. J. Appl. Math. 2012, Article ID 652465 (2012)

24. Su, H, Liu, L, Wang, X: Higher-order dynamic delay differential equations on time scales. J. Appl. Math. 2012, Article ID $939162(2012)$

25. Yolcu, N, Topal, S: Existence of positive solutions of a nonlinear third-order $m$-point boundary value problem for p-Laplacian dynamic equations on time scales. Nonlinear Dyn. Syst. Theory 12, 311-323 (2012)

26. Zhao, J, Lian, H, Ge, W: Existence of positive solutions for nonlinear $m$-point boundary value problems on time scales. Bound. Value Probl. 2012, 4 (2012)

27. Goodrich, CS: The existence of a positive solution to a second-order delta-nabla $p$-Laplacian BVP on a time scale. Appl. Math. Lett. 25, 157-162 (2012)

28. Guo, DJ, Lakshmikantham, V: Nonlinear Problems in Abstract Cones. Academic Press, Boston (1988)

29. Avery, Rl, Henderson, J: Two positive fixed points of nonlinear operators on ordered Banach spaces. Commun. Appl. Nonlinear Anal. 8, 27-36 (2001)

30. Leggett, RW, Williams, LR: Multiple positive fixed points of nonlinear operators on ordered Banach spaces. Indiana Univ. Math. J. 28, 673-688 (1979)

doi:10.1186/1687-1847-2013-206

Cite this article as: Thiramanus and Tariboon: Positive solutions of $m$-point integral boundary value problems for second-order p-Laplacian dynamic equations on time scales. Advances in Difference Equations 2013 2013:206.

\section{Submit your manuscript to a SpringerOpen ${ }^{\ominus}$ journal and benefit from:}

- Convenient online submission

- Rigorous peer review

Immediate publication on acceptance

Open access: articles freely available online

High visibility within the field

- Retaining the copyright to your article 